\title{
Patterns of EEG Frequency, Movement, Heart Rate, and Oxygenation after Isolated Short Apneas in Infants
}

\author{
MARIE-FRANÇOISE VECCHIERINI, LILIA CURZI-DASCALOVA, HA TRANG-PHAM, \\ JULIETTE BLOCH, AND CLAUDE GAULTIER \\ Service de Physiologie, Hopital Bichaty, Université Paris VII, 75877 Paris Cédex 18, France [M.-F.V.]; \\ and Service de Physiologie [L.C.-D., H.T.-P., C.G.], INSERM E 9935, and Service de Santé Publique \\ [J.B.], Hôpital Robert Debré-Université Paris VII, 75019 Paris, France
}

\section{ABSTRACT}

\begin{abstract}
Patterns of events occurring at the end of apneas have rarely been reported in infants. No previous studies have compared these patterns to those of spontaneous events during sleep. We examined 163 isolated apneas in 17 infants $(47 \pm 4$ wk postconceptional age) who underwent polysomnography for suspected upper airway problems. Mean apnea duration was $6.5 \pm 1.5 \mathrm{~s}$ (range, 5 to $11.5 \mathrm{~s}$ ), $78 \%$ of apneas occurred in active sleep, and $67 \%$ of apneas were obstructive. We recorded the occurrence of body movement or augmented breath and analyzed changes in EEG frequency $\geq 1 \mathrm{~s}$, heart rate, and oxygen saturation value at the end of apneas and of a control ventilatory period defined as a period of breathing equal in duration to the apnea and preceding the apnea by $1 \mathrm{~min}$. We found that $7.9 \%$ of apneas and $11.6 \%$ of control periods were followed by an augmented breath and that $14.1 \%$ of apneas and $0.5 \%$ of control periods were followed by a body movement. The percentages of motor events or no event differed significantly after the apneas $(p=0.008)$ compared with the control periods. A significant increase in EEG
\end{abstract}

frequency was observed at the end of the apneas compared with the control periods $(p<0.04)$. EEG frequency increased after $61 \%$ of the apneas. Neither heart rate nor oxygen saturation value changed after the control periods. Heart rate decreased significantly after the apneas not followed by a motor event $(p=0.02)$ but not after the apneas followed by a body movement. We conclude that 1 ) at termination of isolated apneas in infants, a motor event was rare, whereas an EEG frequency increase was common; 2) event patterns at apnea termination differed from those at control period termination. (Pediatr Res 49: 220-226, 2001)
AS, active sleep
Abbreviations
IS, indeterminate sleep
QS, quiet sleep
ALTE, apparently life-threatening event

Events such as awakenings, body movements, and augmented breaths occur spontaneously during sleep in infants (1-5), being apparently related to periodic excitatory mechanisms essential for respiratory homeostasis and normal development $(6,7)$.

Little is known about the events associated with the postapnea recovery phase in infants. Thoppil et al. (8) used video recordings to identify behavioral arousals (awakenings) in apneic preterm infants and found that many apneas were not accompanied with arousal. In a study of EEG arousals in infants, McNamara et al. (9) found that fewer than $8 \%$ of apneas ended by an EEG arousal. These two studies suggest that cortical arousal may not be an important mechanism in the termination of respiratory events in infants.

Received September 15, 1999; accepted June 14, 2000

Correspondence and reprint requests: Marie-Françoise Vecchierini, M.D., Service de Physiologie, Hôpital Bichat, Université Paris VII, 75877 Paris Cédex 18, France.

Supported by grants from Université Paris VII (EA 2379) and INSERM E 9935.
Our study was designed 1) to examine the patterns of events after isolated apneas in infants; and 2) to test the hypothesis that these patterns may be different from nonapnea-related patterns of events occurring during the same sleep state period. We examined the following events: awakening, body movement, augmented breath, changes in EEG frequency, heart rate, and oxygenation at apnea termination. We selected isolated apneas to eliminate possible effects of antecedent events such as apneas or motor activity (10). For each isolated apnea, we determined a ventilatory period equal in length to the apnea and preceding the apnea by $1 \mathrm{~min}$. This period served as the control period; events noted at termination of control periods were considered spontaneous (nonapnea-related).

\section{METHODS}

\section{Patients}

We studied 17 infants referred to our sleep laboratory for polysomnography to evaluate suspected upper airway prob- 
lems during sleep. Nine infants had craniofacial abnormalities (Pierre Robin syndrome, $n=4$; cleft palate, $n=4$; and micrognathia, $n=1$ ), two had laryngomalacia, four had a history of ALTE, and two were referred for clinically documented apneas. None of the infants had neurologic or cardiovascular disorders, and, at the time of the study, none were on medications known to affect sleep states and/or respiratory and cardiac control. Informed consent was obtained from the parents, and the study was approved by the appropriate ethics committee.

\section{Methods}

Polysomnography was performed during an afternoon nap after the usual feed without previous sleep deprivation or sedation. Room temperature was $23^{\circ} \mathrm{C}$. The infants were dressed normally. The two infants with craniofacial abnormalities who usually slept in the prone position were tested in that position; the other 15 infants were tested in the supine position. Each infant was monitored using at least two EEG leads (A2C4, A1C3, according to the 10-20 international system), two electrooculograms, a submental electromyogram, and a precordial ECG lead. Two small actimeters were placed on one hand and the opposite foot, respectively. Nasobuccal airflow was recorded using a thermistance; rib cage and abdominal movements were recorded using strain gauges. Oxygen saturation value $\left(\mathrm{SaO}_{2}\right)$ was measured using a pulse oximeter (NELLCOR 2000) with the sensor placed on a toe. $\mathrm{SaO}_{2}$ sampling was set at $1 \mathrm{~s}$. All these parameters were stored on a digitized polysomnograph (Alice 3, Healthdyne-Respironics). The infants were observed continuously by a trained nurse.

Four behavioral states, namely QS, AS, IS, and awakening, were scored according to the concordance of EEG features and to the presence or absence of eye movements $(11,12)$. Sleep stages were scored by 30-s epochs, but interruptions in state characteristics shorter than 1 min were included in the ongoing state.

Apnea was defined as cessation of respiratory signals for at least $5 \mathrm{~s}$. Obstructive and central apneas were scored according to the American Thoracic Society consensus statement (13). Mixed apneas were included among the obstructive apneas. We analyzed only those apneas not preceded within $10 \mathrm{~s}$ by a motor event (body movement or augmented breath) or an apnea. The number of selected apneas expressed as the apnea index (number of apneas per hour of sleep) and the total apnea indices recorded during polysomnography are indicated in Table 1. Body movements were assessed on the actimeter recording and/or the pulse wave signal of the oximeter trace. Augmented breath at the end of an apnea was defined as a breath with at least twice the mean amplitude of the three breaths preceding the apnea. Computer-assisted EEG frequency measurement was used to calculate EEG frequency changes $\geq 1 \mathrm{~s}$ measured on the same EEG lead during a similar pattern of the same sleep state within the 3-s periods preceding and after each apnea, or as soon as the artifacts sometimes produced by body movements had disappeared. The $\mathrm{SaO}_{2}$ value immediately before each apnea and the lowest value during the $10 \mathrm{~s}$ after the end of the apnea were recorded. Heart
Table 1. Characteristics of the sleep recordings and apneas

\begin{tabular}{lcc}
\hline & Mean and SD & Range \\
\hline Recording time (min) & $158.5 \pm 24.4$ & $(110-210)$ \\
Total sleep time (min) & $120.5 \pm 24.5$ & $(73.5-155)$ \\
AS time (min) & $60.2 \pm 27.7$ & $(18-131)$ \\
QS time (min) & $56.3 \pm 18.6$ & $(21.5-86)$ \\
Apnea index & & \\
$\quad$ On the recording & & \\
$\quad$ CA 3-5 s & $5.4 \pm 5.2$ & $(0-16.7)$ \\
$\quad \geq 5 \mathrm{~s}$ & $4.0 \pm 6.6$ & $(0-22)$ \\
OA 3-5 s & $4.0 \pm 7.7$ & $(0-29.1)$ \\
$\quad \geq 5 \mathrm{~s}$ & $5.1 \pm 8.8$ & $(0-28.3)$ \\
Selected for the study & & \\
CA $\geq 5 \mathrm{~s}$ & $1.55 \pm 2.2$ & $(0-7.4)$ \\
OA $\geq 5 \mathrm{~s}$ & $2.9 \pm 5.5$ & $(0-20.8)$ \\
Mean duration & & \\
CA (s) & $6.1 \pm 1.3$ & $(5-9.5)$ \\
OA (s) & $6.7 \pm 1.5$ & $(5-11.5)$ \\
Longest selected apnea (s) & $8.3 \pm 2.2$ & $(5-11.5)$ \\
Minimal Sa $\%$ in selected apneas & $93.3 \pm 3.3$ & $(85-98)$ \\
\hline
\end{tabular}

rate was calculated as the mean of the instantaneous values recorded during one respiratory cycle immediately before and after each apnea. If a body movement or an augmented breath occurred at the end of the apnea, heart rate values were calculated immediately before the apnea and after the postapnea event.

For each apnea, a ventilatory period equal in duration to the apnea and preceding the apnea by 1 min within the same sleep state was defined, as shown in Figure 1, and used as the control period (14). Body movements, augmented breaths, and changes in EEG, $\mathrm{SaO}_{2}$, and heart rate were evaluated before and after each control period in the same way as before and after each apnea.

EEG, $\mathrm{SaO}_{2}$, and heart rate changes after the apneas and their matched control periods were expressed as the percentage of modification normalized for the value preceding the apneas or

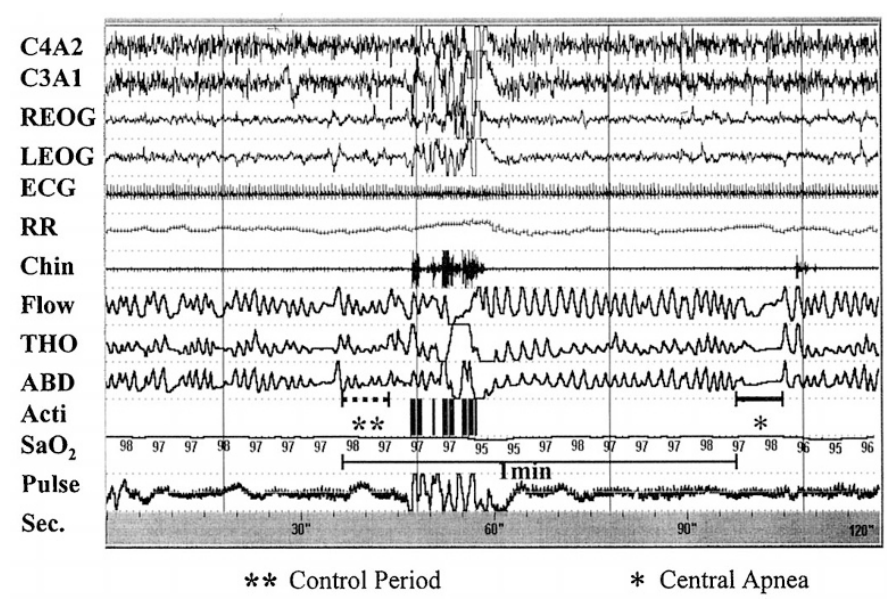

Figure 1. Example of a recording with a central apnea and its control period defined as the period of breathing of equal length as the apnea and preceding the apnea by $1 \mathrm{~min}$. $C 4 A 2$ and $C 3 A 1$ indicate EEG leads; $R E O G$, right eye electrooculogram; $L E O G$, left eye electrooculogram; $R R$, RR interval; Chin, electromyogram of the chin muscles; Flow, nasobuccal flow; THO, thoracic respiration; $A B D$, abdominal respiration; Acti, actimeter; Pulse, oxymetry pulse; Sec., time base in seconds. 
control periods, i.e. as the ratio of the preapnea value minus the postapnea value over the preapnea value, multiplied by 100 . This percentage change was negative if the value increased and positive if it decreased.

For our analysis of changes in EEG frequency, heart rate, and $\mathrm{SaO}_{2}$, we separated the 163 selected apneas into three groups: apneas not ended by a body movement nor by an augmented breath, apneas ended by a body movement, and apneas ended by an augmented breath.

\section{Statistical Analysis}

All values are reported as mean $\pm \mathrm{SD}$. Univariate analysis of quantitative parameters was done using ANOVA. The $\chi^{2}$ test was used to evaluate qualitative variables. In our analyses of paired observations, i.e. apnea versus control periods or before versus after an apnea (or a control period), we used the McNemar test for qualitative variables and a paired parametric test ( $t$ test) or a paired nonparametric test (Wilcoxon test) for quantitative variables, depending on sample size. For each subgroup analysis (apneas not followed by a motor event, apneas followed by an augmented breath, and apneas followed by a movement), Bonferroni's correction was applied.

To look for possible independent effects of apnea duration, apnea type (central or obstructive), sleep state, and postconceptional age on events after apneas, we performed multivariate logistic regression adjusted on subject effect. We constructed a multivariate linear model taking the subject factor as a cluster to identify possible independent effects of the same factors on EEG, heart rate, and $\mathrm{SaO}_{2}$ modifications. $p$ values $\leq$ 0.05 were considered significant.

\section{RESULTS}

\section{Study Infants}

Eleven infants were full-term and six were preterm (mean gestational age was $38 \pm 3.5 \mathrm{wk}$ ). At the time of the study, mean postconceptional age was $47 \pm 4 \mathrm{wk}$ (range, 41 to 56 wk).

\section{Sleep and Apnea Characteristics}

Total recording time, total sleep time, sleep state duration (mean, SD), and characteristics of the 163 apneas selected for analysis are reported in Table 1. Our sample included more obstructive apneas than central apneas, and the obstructive apneas were more numerous in AS than in QS. Only two apneas were recorded in IS; both were excluded from our analysis. The apneas were of short duration (mean, $6.5 \pm$
$1.5 \mathrm{~s}$ ); the longest obstructive apnea lasted $11.5 \mathrm{~s}$. There was no significant relation between apnea duration and sleep state. During QS, mean apnea duration was not significantly different between obstructive and central apneas $(6.7 \pm 1.5$ and $6.7 \pm$ 1.4, respectively). During AS, obstructive apneas were significantly longer $(p<0.01)$ than central apneas $(6.7 \pm 1.4$ and 5.7 \pm 0.9 , respectively).

\section{Motor Events at the End of Apneas and Control Periods}

No motor events occurred after $76.4 \%$ of the apneas; $14.1 \%$ of the apneas were terminated by a body movement, $7.9 \%$ by an augmented breath, and $1.6 \%$ by an awakening. No significant differences were observed in these percentages between obstructive and central apneas or between apneas occurring in AS and QS. Among the matched control periods, 11.6\% ended by body movements, $0.5 \%$ by an augmented breath, and $0.4 \%$ by an awakening; for $87.5 \%$ of control periods, none of these events occurred. The percentages of motor events or no event differed significantly after the apneas and after the control periods $(p=0.008)$.

\section{EEG Frequency, Heart Rate, and $\mathrm{SaO}_{2}$ Changes}

Changes in EEG frequency, heart rate, and $\mathrm{SaO}_{2}$ were analyzed separately in the three groups of apneas. The characteristics of the three groups are shown in Table 2. Mean EEG frequency and mean $\mathrm{SaO}_{2}$ were not significantly different before the apneas versus before the control periods in any of the three groups of apneas. Mean heart rate was significantly different before apneas ended by an augmented breath versus before the control periods $(p=0.03)$. No significant differences were observed in mean EEG frequency, mean heart rate, and mean $\mathrm{SaO}_{2}$ before versus after the control periods in any of the three groups of apneas. Absolute values (mean and range) of EEG frequency, heart rate, and $\mathrm{SaO}_{2}$ before and after apneas and their corresponding control periods in the three groups of apneas are reported in Table 3 .

\section{Apneas Not Ended by a Body Movement or an Augmented Breath}

$\boldsymbol{E E G}$ frequency. Mean EEG frequency was significantly greater after than before the apneas $(p<0.03)$. The mean percentage of EEG frequency acceleration after the apneas $(-8.4 \pm 21.1 \%)$ was significantly greater than after the control periods $(-2.6 \pm 13.5 \%)(p=0.05)$. However, this acceleration in EEG frequency was noted only after $59.7 \%$ of apneas, in a frequency band from 0.1 to $3 \mathrm{~Hz}(-0.7 \pm 0.6 \mathrm{~Hz})$, and the

Table 2. Characteristics of the three groups of selected apneas

\begin{tabular}{|c|c|c|c|}
\hline & $\begin{array}{l}\text { Apnea not ended } \\
\text { by a motor event }\end{array}$ & $\begin{array}{l}\text { Apnea ended by an } \\
\text { augmented breath }\end{array}$ & $\begin{array}{l}\text { Apnea ended by a } \\
\text { body movement }\end{array}$ \\
\hline No. of apneas & 124 & 12 & 27 \\
\hline No. of study infants & 15 & 5 & 8 \\
\hline Mean duration (s) $\pm \mathrm{SD}$ & $6.4 \pm 1.4$ & $7.3 \pm 1.9$ & $6.5 \pm 1.5$ \\
\hline Range & $(5-11.5)$ & $(5-11.5)$ & $(5.5-10)$ \\
\hline$\%$ of $\mathrm{OA}$ & 67 & 58 & 74 \\
\hline$\%$ of apnea in AS & 78 & 83 & 85 \\
\hline
\end{tabular}


Table 3. EEG frequency, heart rate, and $\mathrm{SaO}_{2}$ values before and after selected apneas and their corresponding control periods

\begin{tabular}{|c|c|c|c|c|c|c|}
\hline & \multicolumn{2}{|c|}{$\begin{array}{c}\text { Apneas not ended by a motor } \\
\text { event }\end{array}$} & \multicolumn{2}{|c|}{ Apneas ended by an augmented breath } & \multicolumn{2}{|c|}{ Apneas ended by a body movemen } \\
\hline & Before & After & Before & After & Before & After \\
\hline \multicolumn{7}{|l|}{ EEG frequency $(\mathrm{Hz})$} \\
\hline Apneas & $\begin{array}{l}3.8 \pm 1.2 \\
(1.6-7)\end{array}$ & $\begin{array}{l}4.1 \pm 1.1 \\
(1.9-8)\end{array}$ & $\begin{array}{c}3.7 \pm 1.3 \\
(2-5.7)\end{array}$ & $\begin{array}{l}4.2 \pm 1.65 \\
(2.5-8.6)\end{array}$ & $\begin{array}{l}4.5 \pm 1.5 \\
(2.2-8.1)\end{array}$ & $\begin{array}{l}5.9 \pm 7.2 \\
(2.3-11.0)\end{array}$ \\
\hline Control periods & $\begin{array}{l}4 \pm 1.15 \\
(0.3-8.5)\end{array}$ & $\begin{array}{l}4.1 \pm 1.2 \\
(0.3-8.3)\end{array}$ & $\begin{array}{l}4 \pm 1.2 \\
(2.5-6)\end{array}$ & $\begin{array}{c}4 \pm 1.2 \\
(2.5-5.8)\end{array}$ & $\begin{array}{l}4.6 \pm 1.5 \\
(2.2-7.2)\end{array}$ & $\begin{array}{l}4.6 \pm 1.5 \\
(2.1-7.5)\end{array}$ \\
\hline Apneas & $\begin{array}{l}132.4 \pm 15 \\
(94.7-173.9)\end{array}$ & $\begin{array}{l}127.9 \pm 13.6 \\
(97.3-158)\end{array}$ & $\begin{array}{r}135.7 \pm 9.7 \\
(122-150)\end{array}$ & $\begin{array}{l}131.9 \pm 10.1 \\
(117.1-147.5)\end{array}$ & $\begin{array}{l}136.2 \pm 11.8 \\
(115.2-157)\end{array}$ & $\begin{array}{r}137.2 \pm 15.9 \\
(98-164.4)\end{array}$ \\
\hline Control periods & $\begin{array}{c}131.6 \pm 15.1 \\
(100-175)\end{array}$ & $\begin{array}{r}132.6 \pm 15.6 \\
(98.6-184.6)\end{array}$ & $\begin{array}{c}130 \pm 8 \\
(118.3-146.3)\end{array}$ & $\begin{array}{l}132.6 \pm 9.3 \\
(118.7-146.3)\end{array}$ & $\begin{array}{r}138.5 \pm 10.5 \\
(119-156.5)\end{array}$ & $\begin{array}{r}137.7 \pm 10.5 \\
(120-156.1)\end{array}$ \\
\hline \multicolumn{7}{|l|}{$\mathrm{SaO}_{2}(\%)$} \\
\hline Apneas & $\begin{array}{r}96.7 \pm 2.5 \\
(85-100)\end{array}$ & $\begin{array}{r}95.9 \pm 2.8 \\
(83-100)\end{array}$ & $\begin{array}{r}97.6 \pm 2.2 \\
(94-100)\end{array}$ & $\begin{array}{r}96.3 \pm 2.6 \\
(93-100)\end{array}$ & $\begin{array}{r}97.1 \pm 2.9 \\
(91-100)\end{array}$ & $\begin{array}{r}96.1 \pm 3.1 \\
(89-100)\end{array}$ \\
\hline
\end{tabular}

* Mean value $\pm \mathrm{SD}$ with range in brackets, control period (see text for definition); $\mathrm{SaO}_{2}$, oxygen saturation (pulse oximeter).

mean percentage of EEG frequency acceleration after the apneas was $-20.1 \pm 19.3 \%$. EEG frequency remained unchanged after $6.5 \%$ of the apneas. In the remaining $33.9 \%$, EEG frequency decreased, the mean percentage of EEG frequency deceleration being $10 \pm 6.9 \%$ (from 0.1 to $1.4 \mathrm{~Hz}$ ). The percentage of EEG frequency change was not related to apnea type, apnea duration, or sleep states.

Heart rate. Mean heart rate was significantly lower after than before the apneas $(p<0.0001)$ and after the apneas than after the control periods $(p<0.0002)$. The mean percentage of heart rate change after the apneas $(3 \pm 7 \%)$ was significantly different from the mean percentage change after the control periods $(-0.9 \pm 6.3 \%)(p<0.0001)$. Heart rate deceleration was found after $70.2 \%$ of the apneas and ranged from 1 to 54 beats per minute $(9 \pm 7.6)$ and from 0.6 to $31 \%(6.2 \pm 5 \%)$. Heart rate remained stable after $4 \%$ of the apneas and increased slightly $(-5.4 \pm 4.9 \%)$ after $25.2 \%$. The mean percentage of heart rate deceleration was not correlated with apnea type or duration, although there was a trend for greater deceleration after longer apneas. A significant relationship was found between the percentage of heart rate deceleration and sleep states $(p<0.004)$ with greater deceleration in AS than in QS.

$\mathrm{SaO}_{2} \cdot \mathrm{SaO}_{2}$ was slightly but significantly lower after the apneas. The mean percentage of $\mathrm{SaO}_{2}$ decrease after the apneas $(0.8 \pm 1.1 \%)$ was significantly greater $(p<0.001)$ than the mean percentage of $\mathrm{SaO}_{2}$ decrease after the control periods $(0.6 \pm 9.1 \%) . \mathrm{SaO}_{2}$ decreased after $52.5 \%$ of the apneas and remained unchanged in the remaining cases. $\mathrm{An} \mathrm{SaO}_{2}$ decrease $>4 \%$ was found only after three central apneas. The percentage of $\mathrm{SaO}_{2}$ decrease after the apneas was significantly related to apnea type $(p=0.0001)$, being larger after central apneas than obstructive apneas, and to apnea duration $(p=0.003)$, being larger with apneas $>7 \mathrm{~s}$. The percentage of $\mathrm{SaO}_{2}$ decrease was not influenced by sleep states.

\section{Apneas Followed by an Augmented Breath}

$\boldsymbol{E} \boldsymbol{E} \boldsymbol{G}$ frequency. A slight nonsignificant $\mathrm{EEG}$ frequency increase was noted after these apneas. The mean percentage of
EEG frequency acceleration after the apneas followed by an augmented breath $(-17.6 \pm 26 \%)$ was significantly greater $(p$ $=0.03)$ than the mean percentage of EEG frequency change after the control periods $(0.15 \pm 10.8 \%)$. EEG frequency acceleration was noted after nine apneas. The percentage of EEG frequency acceleration was not related to apnea type or duration.

Heart rate. The heart rate decrease after the apneas was not statistically significant. The mean percentage of heart rate change was not significantly different after the apneas $(2.7 \pm$ $13.9 \%)$ and after the control periods $(2.1 \pm 6.3 \%)$. Heart rate deceleration was found after seven apneas. The percentage of heart rate change after the apneas was not related to apnea duration or type.

$\mathrm{SaO}_{2} \cdot \mathrm{SaO}_{2}$ decreased significantly after the apneas $(p<$ $0.02)$. The mean percentage of $\mathrm{SaO}_{2}$ decrease after the apneas $(1.3 \pm 1.6 \%)$ was significantly different $(p=0.003)$ from the mean percentage after the control periods $(-0.3 \pm 0.7 \%)$. $\mathrm{SaO}_{2}$ decreased after $50 \%$ of the apneas and remained unchanged in the other cases. The $\mathrm{SaO}_{2}$ decrease percentage was significantly ( $p<0.01)$ related to apnea duration (only for apneas longer than $7 \mathrm{~s}$ ) but was not correlated to apnea type.

\section{Apneas Followed by a Body Movement}

EEG frequency. The increase in EEG frequency after the apneas was not statistically significant. However, the mean percentage of EEG frequency acceleration after the apneas $(-33.8 \pm 1.6 \%)$ was significantly greater $(p<0.01)$ than the mean percentage after the control periods $(-2.4 \pm 14.1 \%)$. EEG frequency acceleration was noted after $59.2 \%$ of the apneas and EEG frequency deceleration after the remaining apneas. The percentage of EEG frequency acceleration was unrelated to apnea duration and postapnea body movement duration but was significantly related to apnea type, being greater after obstructive apneas $(p<0.05)$.

Heart rate. Mean heart rate did not differ significantly before and after apneas followed by a body movement. There was a slight increase in mean heart rate after the apneas. The mean 
percentage of heart rate acceleration after the apneas was not significantly different $(-1.1 \pm 11.8 \%)$ from the mean percentage change after the control periods $(0.5 \pm 5.3 \%)$. Heart rate increased after 12 apneas, remained unchanged after one, and decreased after 14 . The percentage of heart rate variation after the apneas was not related to apnea type, apnea duration, or body movement duration.

$\mathrm{SaO}_{2}$. Mean $\mathrm{SaO}_{2}$ decreased significantly after the apneas ( $p$ $=0.002)$. The mean percentage of $\mathrm{SaO}_{2}$ decrease after the apneas $(3.6 \pm 19.3 \%)$ was significantly different from the mean percentage after the control periods $(-1 \pm 1.5 \%)(p<$ $0.001) . \mathrm{SaO}_{2}$ decreased after 17 apneas. The percentage of $\mathrm{SaO}_{2}$ change was significantly related to apnea duration $(p<$ 0.01 ) but not to apnea type or body movement duration.

\section{Comparison Between EEG Frequency, Heart Rate, and $\mathrm{SaO}_{2}$ Changes Within and Between the Three Groups of Apneas}

There was no significant relationship between the mean percentage changes in EEG frequency, heart rate, and $\mathrm{SaO}_{2}$ after the apneas in any of the three groups.

The comparison of the mean percentage of postapnea EEG frequency change in the three groups of apneas showed no significant differences, although the EEG frequency increase tended to be larger after apneas followed by a body movement. For the three groups combined, $61 \%$ of apneas were followed by an increase in EEG frequency, and the mean percentage of EEG frequency increase was significantly different after the apneas compared with after the control periods $(p<0.04)$.

The mean percentage of $\mathrm{SaO}_{2}$ change decreased after the apneas in each group with no significant differences across the three groups.

The mean percentage of heart rate change decreased after the apneas not followed by a motor event and after those followed by an augmented breath with no significant difference between these two groups. However, the mean percentage of heart rate variation increased after the apneas followed by a body movement, and there was a significant difference between the mean heart rate changes after the apneas followed by no motor event and after the apneas followed by a body movement $(p=0.02)$, as shown in Figure 2.

Postconceptional age did not influence changes in EEG frequency, heart rate, and $\mathrm{SaO}_{2}$ in any group of the three apneas.

\section{DISCUSSION}

The aims of the present study were to examine the patterns of events after isolated apneas in infants and to test the hypothesis that these patterns may be different from spontaneous events occurring during the same sleep state. We found that both central and obstructive isolated apneas were rarely ended by an augmented breath or a body movement, but that both events were significantly more common after apneas than after matched control periods. A significant increase in EEG frequency was observed at the end of the apneas compared with the end of the matched control periods. No significant changes in heart rate or in oxygenation were observed after the

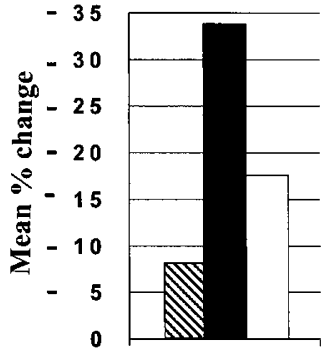

EEG frequency

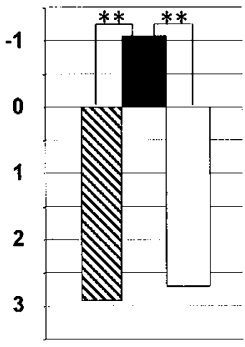

Heart rate

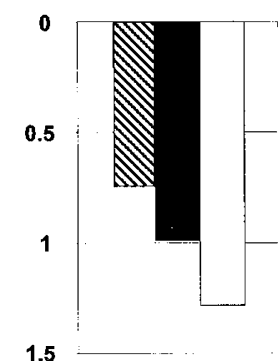

$\mathrm{SaO}_{2}$
Figure 2. Mean percentage of changes in EEG frequency, heart rate, and $\mathrm{SaO}_{2}$ after the apneas. See "Methods" for the calculation of the mean percentage of change. This percentage was negative if the value increased and positive if it decreased. The gray columns represent the apneas not followed by a motor event; the black columns, the apneas followed by a body movement, and the open columns the apneas followed by an augmented breath. The mean percentage change in heart rate was significantly different after the apneas followed by no motor event $v s$ after the apneas followed by a body movement $(p<0.02)$.

control periods. Apneas not followed by a body movement were associated with a slight but significant heart rate decrease, whereas heart rate did not change significantly at the end of apneas followed by a body movement.

\section{Methodologic Considerations}

Events preceding apneas can influence the characteristics of apneas $(10,15)$. We, therefore, analyzed isolated apneas not preceded by a body movement. The selected apneas were more frequent in AS than in QS. Obstructive apneas were more frequent than central apneas in our study of infants suspected of upper airway problems during sleep.

Because oscillations periodically occur during sleep and may affect cortical and sympathetic system influences controlling heart rate and motor activity $(4,6,7)$, we attempted to specifically characterize events related to apnea termination. We consequently examined a control ventilatory period of equal duration as each apnea and preceding the apnea by $1 \mathrm{~min}$ within the same sleep state. Our setup did not include esophageal pressure measurements. Therefore, the possibility exists that increased esophageal pressure variations during the minute preceding the analyzed apneas may have been responsible for the EEG frequency increase (16). Nevertheless, it is important to point out that we found, on average, no significant differences in EEG frequency, heart rate, or $\mathrm{SaO}_{2}$ before the apneas and before their matched control periods. This argues against major changes in respiratory efforts during the minute preceding the selected apneas. Furthermore, the mean percentage of change in EEG frequency was significantly greater at the end of all apneas than at the end of the matched control periods.

\section{Motor Activity at the End of Apneas}

The majority of the short isolated apneas terminated without motor activity, either a body movement or an augmented breath. Augmented breaths were infrequent both after the control periods $(0.5 \%)$ and after the apneas (less than $10 \%$ ). Body movements at apnea termination were rare, occurring 
with nearly the same frequency as after the control periods. Because the majority of the apneas followed by a body movement occurred during AS, it is not surprising that the percentage of control periods and apneas followed by a body movement were similar $(1,3)$. Most of the apneas followed by a body movement were obstructive. This finding is in agreement with a previous study by Hoppenbrouwers et al. (15). The low frequency of motor activity after short isolated apneas as well as after control periods in our study may be ascribable to experimental factors. Two of our study infants were tested in the prone position, and it has been shown that arousals are less common in the prone position than in the supine position (17, 18). Another possibly relevant fact is that four infants had a history of ALTE: it has been reported that infants who subsequently died of sudden infant death syndrome had low motor activity during sleep (19). Finally, some of our study infants had craniofacial abnormalities, which may have been associated with decreased arousability as reported in infants with obstructive sleep apnea syndrome (20).

\section{Changes in EEG Frequency, $\mathrm{SaO}_{2}$, and Heart Rate at the End of Apneas}

Because both body movements (21) and augmented breath (22) interact with changes in heart rate, we separately analyzed EEG frequency, heart rate, and $\mathrm{SaO}_{2}$ changes after apneas of each group. In all three apnea groups, mean EEG frequency was higher after than before the apneas. Approximately $60 \%$ of apneas in all three groups ended by an EEG frequency increase. EEG frequency increase is the most frequent EEG pattern of cortical arousal in adults $(23,24)$. However, there is no clear definition of EEG arousal in infants. An EEG frequency shift $\geq 1 \mathrm{~s}$ has been chosen as one of the criteria used to define movement arousal at the end of apneas in children (25). McNamara et al. (9) used the criteria for EEG arousal recommended in adults (23) but reduced the shift duration from $>3$ to $>1 \mathrm{~s}$. Thus, the minimal EEG change used to indicate cortical arousal remains arbitrary in infants. Nevertheless, frequent episodes of cortical arousal may disturb sleep, especially during AS in infants (26).

A slight but significant $\mathrm{SaO}_{2}$ decrease was found after apneas but not after control periods. The $\mathrm{SaO}_{2}$ decrease was significantly related to the duration of apneas followed by no motor event.

Heart rate did not change significantly before versus after the control periods. Mean heart rate decreased slightly but significantly after the apneas not followed by a motor event or followed by an augmented breath. Interestingly, apneas ended by a body movement were not followed by a significant change in heart rate, although heart rate tended to increase. Sympathetic drive contingent on somatic activity may have overridden the deceleration observed in the other two groups of apneas. An increase in heart rate associated with a body movement with or without an EEG frequency shift has been described as part of movement arousal at the end of obstructive apneas in infants (15) and in children $(25,27)$.

In summary, short isolated apneas were rarely ended by a motor event. However, the percentage of occurrence of a motor event or no event differed significantly after the apneas and after their matched control periods. The occurrence of a body movement at the end of an apnea appeared to prevent the heart rate deceleration observed when somatic motor activity was lacking. Overall, $61 \%$ of the apneas ended with an increase in EEG frequency consistent with cortical arousal. Finally, 39\% of short isolated apneas terminated without any identified event. This finding invites questions about the mechanisms that terminate those apneas in infants. The most likely explanation is that the hypoxic threshold for arousal was not reached during those apneas. Breathing may resume because of other excitatory influences, especially during AS because this state is characterized by instability of central neural respiratory control. The clinical message of our findings is that cortical arousal as assessed by an increase in EEG frequency can occur even after short isolated apneas in infants. Although the point at which EEG changes become physiologically significant remains to be determined in infants, consensus criteria are needed to determine the natural arousal pattern and its change with development and sleep-disordered breathing in infants.

\section{REFERENCES}

1. Curzi-Dascalova L, Plassart E 1978 Respiratory and motor events in sleeping infants: their correlation with thoraco-abdominal relationships. Early Hum Dev 2:39-50

2. Schultz H, Massetani R, Fagioli G, Salzarulo P 1985 Spontaneous awakening from sleep in infants. Electroencephal Clin Neurophysiol 6:267-271

3. Vecchierini-Blineau MF, Nogues B, Louvet S, Desfontaines O 1994 Maturation de la motilité généralisée, spontanée au cours du sommeil, de la naissance à terme à l'âge de 6 mois. Neurophysiol Clin 24:141-154

4. Lijowska AS, Reed NW, Mertins Chiodini BA, Thach BT 1997 Sequential arousal and airway-defensive behavior of infants in asphyxial sleep environments. J Appl Physiol 83:219-228

5. McNamara F, Wulbrand H, Thach BT 1998 Characteristics of the infant arousal response. J Appl Physiol 85:2314-2321

6. McGinty DJ, London MS, Baker TL, Stevenson M, Hoppenbrouwers TH, Harper RM, Sterman MB, Hodgman J 1979 Sleep apnea in normal kittens. Sleep 1:393-421

7. Orem J, Trotter H 1993 Medullary respiratory neuronal activity during augmented breaths in intact unanesthetized cats. J Appl Physiol 74:761-769

8. Thoppil CK, Belan MA, Cowen CP, Mathew OP 1991 Behavioral arousal in newborn infants and its association with termination of apnea. J Appl Physiol 70:2479-2484

9. McNamara F, Issa FG, Sullivan CE 1996 Arousal pattern following central and obstructive breathing abnormalities in infants and in children. J Appl Physiol $81: 2651-2657$

10. Weintraub Z, Alvaro R, Mills S, Cates D, Rigatto H 1994 Short apneas in their relationship to body movements and sighs in preterm infants. Biol Neonate 66:188194

11. Guilleminault C, Souquet M 1979 Sleep states and related pathology. In Korobkin R, Guilleminault C (eds) Advance in Perinatal Neurology. SP Medical and Scientific Book, New York, pp 225-247

12. Curzi-Dascalova L, Mirmiran M 1996 Manual of Methods of Recording and Analyzing Sleep-Wakefulness States in Preterm and Full-term Infants. INSERM, Paris, pp $1-162$

13. American Thoracic Society 1996 Standards and indications for cardiopulmonary sleep studies in children. Am J Respir Crit Care Med 153:866-878

14. Curzi-Dascalova L, Bloch J, Vecchierini MF, Bedu A, Vignolo P 2000 Physiological parameters evaluation following apnea in healthy premature infants. Biol Neonat 77:203-212

15. Hoppenbrouwers T, Hodgman JE, Cabal L 1993 Obstructive apnea, associated patterns of movement, heart rate, and oxygenation in infants at low and increased risk for SIDS. Pediatr Pulmonol 15:1-12

16. Guilleminault C, Pelayo R, Leger D, Clerk A, Bocian CZ 1996 Recognition of sleep-disordered breathing in children. Pediatrics 98:871-882

17. Kahn A, Groswasser J, Sottiaux M, Franco P, Dramaix M 1993 Prone and supine body position and sleep characteristics in infants. Pediatrics 91:1112-1115

18. Franco P, Groswasser J, Sottiaux M, Broadfield E, Kahn A 1996 Decreased cardiac responses to auditory stimulation during prone sleep. Pediatrics 97:174-178

19. Kahn A, Groswasser J, Rebuffat E, Sottiaux M, Blum D, Foerster M, Franco P, Bochner A, Alexander M, Bachy A 1992 Sleep and cardiorespiratory characteristics of infant victims of sudden death: a prospective case-control study. Sleep 15:287-292

20. McNamara F, Sullivan CE 1999 Effects of nasal CPAP therapy on respiratory and spontaneous arousals in infants with OSA. J Appl Physiol 87:889-896

21. Curzi-Dascalova L, Kauffmann F, Gaultier C, Caldas de Amorim RH 1999 Heart rate modifications related to spontaneous body movements in sleeping premature and full-term newborns. Pediatr Res 45:515-518 
22. Eiselt M, Curzi-Dascalova L, Leffler CH, Christova E 1992 Sigh-related heart rate changes during sleep in premature and full-term newborns. Neuropediatrics 23:286291

23. EEG arousals scoring rules and examples 1992 A preliminary report from the sleep disorders atlas task force of the American Sleep Disorders Association. Sleep 15:174-184

24. Associazione Italiana di Medicina del Sonno 1997 Phasic event appearing in all stages of sleep. In: Terzano MG, Parrino L, Mennuni GF (eds) Phasic Events and Microstructure of Sleep. Martano, Lecce, pp 114-120
25. Mograss MA, Ducharme FM, Brouillette RT 1994 Description, classification, and relationship to sleep apnea in children. Am J Respir Crit Care Med 150:16901696

26. McNamara F, Sullivan CE 1999 Obstructive sleep apnea in infants and its management with nasal continuous positive airway pressure. Chest 116:10-16

27. Praud JP, D'Allest AM, Nedelcoux H, Curzi-Dascalova L, Guilleminault C, Gaultier C 1989 Sleep-related abdominal muscle behavior during partial or complete obstructed breathing in prepubertal children. Pediatr Res 26 $347-350$ 